Stefan Kubica, Hagen Ringshausen, Jörg Reiff-Stephan, Marius Schlingelhoff (Hrsg.) 1. Automobil Symposium Wildau: Tagungsband Technische Hochschule Wildau 2016
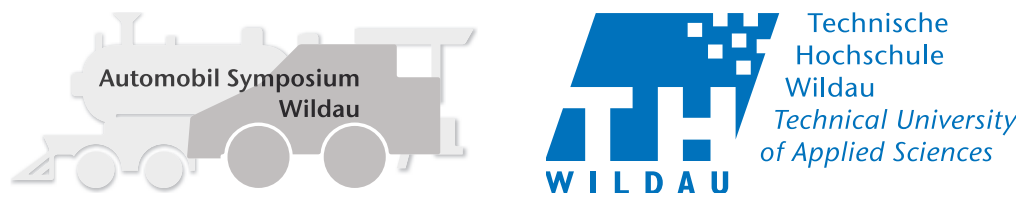

\title{
Car2X-Kommunikation als Grundlage für Effizienz- und Assistenzfunktionen für den Verkehr der Zukunft
}

\section{Zusammenfassung}

Fahrzeughersteller und Politik haben in den letzten Jahrzehnten durch konsequentes Handeln die Getöteten im Straßenverkehr erheblich reduziert und gleichzeitig dafür gesorgt, dass unsere Mobilität umweltschonender und auch komfortabler wurde. Im Vergleich zu den 1970er Jahren hat sich die Verkehrsleistung verdreifacht - im gleichen Zeitraum ging die Anzahl der Getöteten im Straßenverkehr jedoch um $80 \%$ zurück.

In Zeiten zunehmender Urbanisierung verändern sich Verkehr und auch das Mobilitätsverhalten.

Zukünftige Fahrzeugfunktionen müssen dem Rechnung tragen. So kommt es insbesondere in Städten und Ballungsräumen zu einem erhöhten Verkehrsaufkommen. Intelligente Effizienzfunktionen arbeiten mit der Infra- struktur Hand in Hand und die Fahrzeuge kooperieren untereinander, um den Verkehr auch zukünftig flüssig zu halten und Lärm und Emissionen weiter zu senken. Es ergeben sich dabei auch Assistenzfunktionen mit Aspekten der Verkehrssicherheit - besonders im urbanen Umfeld steht dabei die Vermeidung von Unfällen im Vordergrund. Um diese Ziele zu erreichen, ist die Einführung neuartiger Technologien notwendig.

Die Car2X-Kommunikation bietet dabei die Möglichkeit des Austauschs von Informationen, sowohl zwischen Fahrzeugen, als auch zwischen Fahrzeugen und der Infrastruktur und bildet damit die Grundlage für ein vernetztes, intelligentes Verkehrssystem. In diesem Paper wird die Car2X-Technologie mit inren Anforderungen für eine Einführung vorgestellt und Vorschläge für die Umsetzung des intelligenten Verkehrssystems im urba-

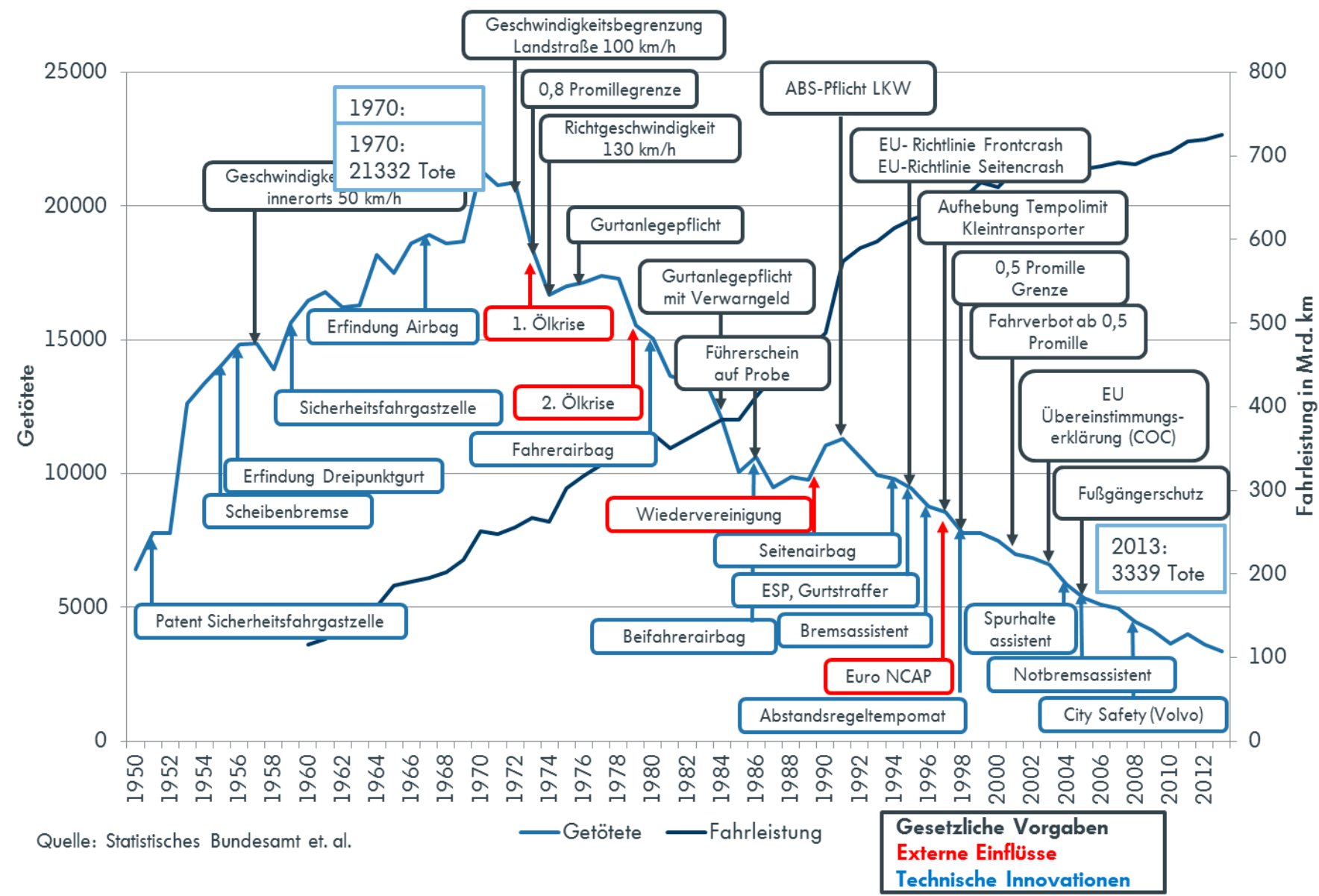

Abb. 1 Entwicklung von Fahrleistung und Getötetenzahlen in Deutschland 
nen Umfeld gegeben. Darüber hinaus werden Funktionen vorgestellt, die Car2X-Kommunikation nutzen, um das Fahren in der Stadt effizienter, komfortabler und sicherer zu gestalten. Dabei werden zunächst Funktionen vorgestellt, die helfen, den Verkehrsfluss zu verbessern und Emissionen zu senken, indem sie Informationen intelligenter Lichtsignalanlagen für die Längsregelung nutzen und damit kooperativ werden. Im Anschluss werden Fahrzeugfunktionen vorgestellt, die einen Beitrag zum sichereren Fahren in der Stadt leisten.

\section{Einführung in die Fahrzeugsicherheit}

Seit Beginn der motorisierten Mobilität um das Jahr 1900 unterlag selbige einem stetigen Wandel. Ab den 1950er Jahren hat sich das Automobil als Mobilitätsmittel für die Masse entwickelt. Damit ging auch ein stetiger, weiterer Anstieg der Verletzten und Getöteten einher. Bereits zu dieser Zeit haben Automobilhersteller wie auch Politik versucht, durch Verbesserung der Sicherheit im Verkehr die Anzahl der Geschädigten zu begrenzen. Trotzdem hat die Anzahl der Getöteten im Straßenverkehr im Jahr 1970 mit 21332 ihren Höhepunkt erreicht. Die Fahrleistung des motorisierten Individualverkehrs betrug dabei lediglich 251 Mrd. km. Im Jahr 2013 gab es dagegen nur noch 3339 Getötete im Straßenverkehr - bei einer Fahrleistung von 725 Mrd. km (Radke 2013).

Damit führt eine Betrachtung der Getöteten bezogen auf die Fahrleistung darauf, dass eine Reduzierung von 85 Getöteten/Mrd. km im Jahr 1970 auf eine Quote von 4,6 Getöteten/Mrd. km in 2013 bereits eine Absenkung der Getötetenquote von $93 \%$ mit sich bringt. In Abb. 1 ist die Entwicklung der Getötetenzahlen, sowie der Verkehrsleistung über der Zeit aufgetragen. Verbunden damit sind die politischen Entscheidungen zur Gesetzgebung im Verkehrsbereich, sowie die technischen Innovationen der Automobilindustrie dargestellt. Daraus ist abzuleiten, welche Maßnahmen zu einer Reduzierung der Getötetenzahlen geführt haben. Die Maßnahmen können dabei nicht losgelöst voneinander betrachtet werden und bedingen einander. Dabei wird der Zusammenhang zwischen Fahrer, Fahrzeug und Fahrumgebung als 3F-Parameterraum bezeichnet (Pion et al. 2012). Nur eine gemeinsame Betrachtung der Parameter kann dazu führen, die Gesamtsicherheit im Verkehr zu verbessern. Verkehrserziehung, die sichere Gestaltung von Straßen und Maßnahmen der passiven und aktiven Sicherheit in Kraftfahrzeugen gehen Hand in Hand, um Verkehrsteilnehmer vor Unfällen und deren Folgen zu schützen.

Im Fokus dieses Beitrags steht die Entwicklung der Sicherheit von Kraftfahrzeugen, die in der Abb. 1 als technische Innovationen in blau dargestellt sind. Im späteren Verlauf zeigt sich jedoch auch hier, dass große Stellhebel nur noch in Kombination mit Maßnahmen der Infrastruktur möglich sind. Durch die oben aufgezeigte massive Senkung der Getöteten in den letzten Jahrzehnten haben sich die Wirkfelder - die durch eine neue Sicherheitsfunktion theoretisch adressierbaren Geschädigten
- verkleinert. Unter dem Strich sind die Stellhebel einzelner Maßnahmen kleiner geworden. Dabei ist eine Betrachtung der Verteilung aktueller Getötetenzahlen hinsichtlich Straßenklasse und Verkehrsmode interessant und zeigt bereits erste Möglichkeiten zur Ableitung von Maßnahmen und gibt eine Einschätzung dazu, wie häufig geäußerte politische Vorschläge wirken könnten.

Ein Beispiel dafür ist die Einführung eines Tempolimits auf Bundesautobahnen. Selbst wenn, stark vereinfachend, davon ausgegangen würde, dass damit alle getöteten PKW-Insassen auf Bundesautobahnen vermieden werden, läge das Wirkfeld nur bei ca. $6 \%$, wie aus Abb. 2 hervor geht. Wird dabei hinzugezogen, dass ca. $40 \%$ der BAB-Strecken bereits geschwindigkeitsbegrenzt sind, sinkt das Wirkfeld auf ca. 3,5 \%. Die Darstellung kann dabei keinen Rückschluss auf die Fahrleistung zulassen, die auf Autobahnen anfällt. Schon heute ist dabei die Autobahn jedoch mit 1,6 Getöteten/Mrd. km nochmals sicherer als der gezeigte Durchschnitt (ADAC 2015).

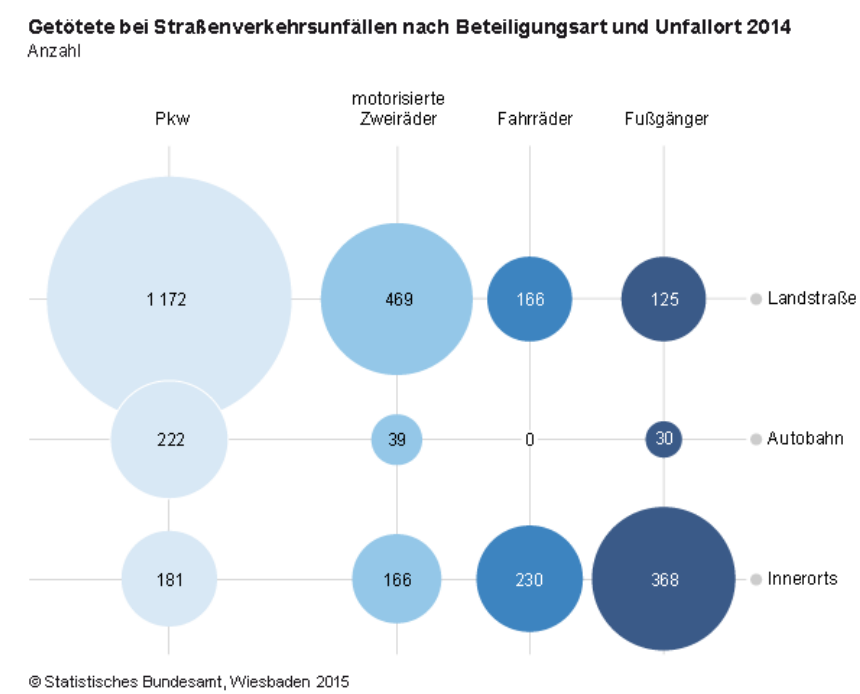

Abb. 2 Verteilung der Getöteten im Straßenverkehr nach Straßenklasse und Verkehrsmode (Statistisches Bundesamt 2015)

Dagegen kamen im Jahr 2014644 Personen bei Baumunfällen ums Leben, die politisch weniger Beachtung finden. Baumunfälle werden häufig durch komplexe Gestaltung, hauptsächlich von Landstraßen, begünstigt. In Flächenbundesländern erreicht der Anteil der Getöteten durch Baumunfälle nahezu $40 \%$ der insgesamt Getöteten im Straßenverkehr (Bakaba \& Kühn 2009). Systeme zur Vermeidung von Baumunfällen hätten demnach ein größeres Wirkfeld als ein Tempolimit.

Passive Maßnahmen haben bereits einen erheblichen Beitrag zur Senkung der Getötetenzahlen geliefert: Baumunfälle enden heute weniger oft tödlich als früher. Maßnahmen zur Senkung der Unfallfolgen, bezeichnet als passive Sicherheit, bilden die Grundlage der Sicherheit von Kraftfahrzeugen. Diese finden sich bereits auch als früh eingeführte technische Innovationen in der oben gezeigten Abb. 1 wieder.

Auch heute bilden die passiven Schutzmaßnahmen weiterhin das Grundgerüst der Fahrzeugsicherheit um den Insassen den nötigen Schutz zu bieten, wenn ein Unfall 


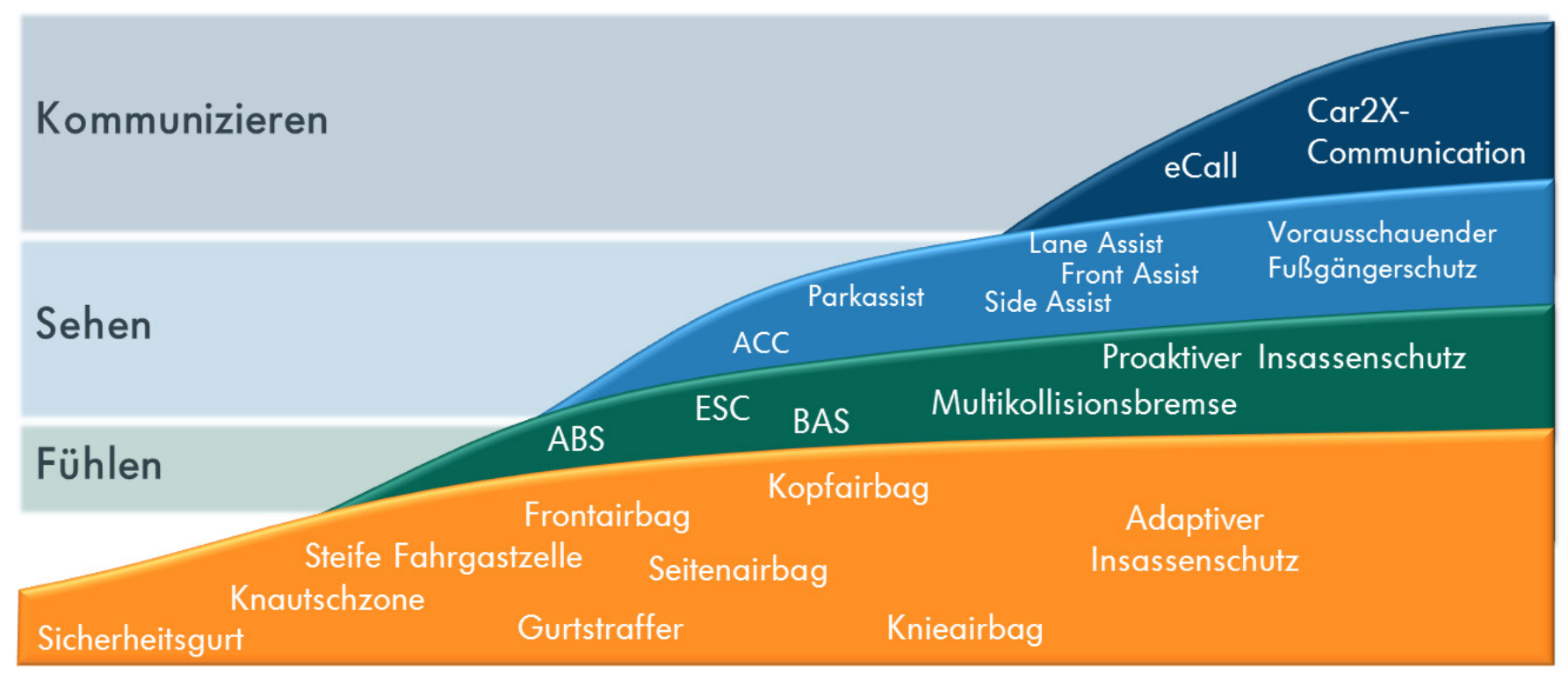

Zeit

Abb. 3 Schutzpotentialgrafik der Fahrzeugsicherheit

nicht mehr vermieden werden kann. In Abb. 3 sind diese im orangenen Bereich dargestellt. Neben der Reduzierung der Unfallfolgen steht vermehrt das Ziel, Unfälle zu verhindern, oder die Schwere von Unfällen zu vermindern, im Fokus der Entwicklung. Hier wird von Maßnahmen der aktiven Sicherheit gesprochen, die in Abb. 3 im grünen und hellblauen Bereich dargestellt sind.

Allen Maßnahmen ist gemein, dass eine Erfassung der Umwelt zu Grunde liegt, auf Basis derer Regelsysteme in den weiteren Fahrtverlauf eingreifen können, oder den Fahrer auffordern zu reagieren. Die Komplexität dieser Sensorsysteme hat dabei kontinuierlich zugenommen. Erste aktive Sicherheitssysteme haben sich auf die Fahrwerkregelung beschränkt. Hier ist das Anti-Blockier-System (ABS) das beste Beispiel. Durch Messung der Raddrehzahl wird durch eine Regelung des Bremsdrucks dafür gesorgt, dass das Fahrzeug seine maximale Verzögerung erreicht und trotzdem lenkfähig bleibt. Im nächsten Schritt haben sich Sensorikkonzepte etabliert, die es erlauben, nicht nur die Bewegungen des Fahrzeugs, sondern auch das Umfeld mit einer größeren Reichweite zu erfassen. Dadurch wird ein Reaktionszeitraum geschaffen und Gefahren werden frühzeitig erfasst. Zu dieser Kategorie gehören beispielsweise Notbremsassistenten, die im Falle eines drohenden Auffahrunfalls das Fahrzeug bei ausbleibender Fahrerreaktion autonom abbremsen. Nach Einführung dieser auf Radar- und/oder Kameraerfassung basierenden Systeme ist die Anzahl der Auffahrunfälle um $38 \%$ zurückgegangen (Fildes et al. 2015) - für den Volkswagen Golf VII weist Thatcham sogar $45 \%$ weniger durch den Golf verursachte Verletzungen im Vergleich zur Wettbewerbsgruppe im britischen Markt aus (Thatham Research 2015). Systeme auf Basis von Radar und Kamera bieten auch Funktionen zur Fußgänger- und Radfahrererkennung. In Abb. 2 nimmt die Gruppe dieser vulnerable road users (VRU), wie dargestellt, bereits rund ein Drittel der insgesamt im Verkehr getöteten Personen ein, entsprechend groß ist das
Wirkfeld. Notbremsassistenten mit Fußgänger- und Radfahrererkennung können einen Beitrag dazu leisten, Kollisionen mit diesen Verkehrsteilnehmern zu reduzieren. Die genannten Sensoren sind auf eine Sichtverbindung zum Messobjekt angewiesen, um verlässliche Ergebnisse zu liefern. Situationen mit Verdeckungen, stark eingeschränkter Sicht durch Nebel oder Schnee sowie Szenarien an Kreuzungen oder an Kuppen sind durch heutige Sensorik nicht vollständig erfassbar. Kommunikation erweitert den Horizont des Fahrzeugs, da zwischen Sender und Empfänger keine Sichtverbindung bestehen muss, zugehörige Funktionen sind in Abb. 3 im dunkelblauen Bereich dargestellt.

2. Kommunikation als neuartige Technologie zum Informationsaustausch im Straßenverkehr

Kommunikation bietet die Möglichkeit eines direkten Informationsaustauschs. Zur Gewinnung von Informationen über die Umgebung ist keine passive Beobachtung über Sensoren notwendig, sondern andere Teilnehmer versenden aktiv Informationen über sich selbst. So lassen sich auch jene Situationen adressieren, die heute von klassischer Umfeldsensorik auf Grund von Verdeckungen nicht erfasst werden können.

\subsection{Grundlagen der Car2X Kommunikation und Standardisierung}

Zum Austausch der Informationen hat sich über die Automobilindustrie hinaus in Zusammenarbeit mit öffentlichen Einrichtungen, Zulieferern und Gremien ETSI ITS G5 als Standard etabliert. Dieser wurde vom European Telecommunication Standardisation Institute (ETSI) im Bereich Intelligent Transportation Systems (ITS) festgelegt. Vereinfacht handelt es sich hierbei um Car2X-Kommunikation über WLANp (Wireless Local Area Network - Substandard $p$ ). Car2X-Kommunikation bezeichnet dabei 
den Austausch von Informationen zwischen Fahrzeugen (Car2Car) und zwischen Fahrzeugen und der Infrastruktur (Car2Infrastructure). WLANp, genauer IEEE 802.11p ist eine Modifikation des bekannten WLAN-Standards, der es Teilnehmern erlaubt, direkt untereinander Informationen ohne einen Access-Point auszutauschen, sobald sie sich in Reichweite befinden. Das Frequenzband liegt bei $5,9 \mathrm{GHz}$ und die Kommunikationsreichweite liegt unter Berücksichtigung der Umgebungsbedingungen bei 100-800 m.

Viele der genannten Teilnehmer sind Partner im Car2Car-Communication-Consortium. Dieses Industriekonsortium bereitet die Standardisierung vor und sorgt darüber hinaus dafür, dass Mechanismen zum Informationsaustausch über alle Hersteller gleich behandelt werden und damit eine Interoperabilität gewährleistet hält dabei zusätzliche Informationen über die Art eines Ereignisses oder einen definierten Zielraum, um etwa Empfänger über ein Ereignis zu benachrichtigen, die sich auf einer ganz bestimmten Strecke dem Sender nähern. Der Standard bringt dabei auch herstellerübergreifende Regeln zur Entstehung der DENM mit, damit ist gewährleistet, dass die Nachrichten aller Quellen in gleichem Maße verlässlich sind und unter identischen Ausgangsbedingungen generiert wurden.

Bis zur Standardisierung waren viele gemeinsame Projekte und Abstimmungen notwendig. Das Prinzip des gemeinsamen Sensierens von Informationen und die Notwendigkeit einer Beteiligung von Sender und Empfänger bringt völlig neue Herausforderungen in der Entwicklung mit sich. Zum ersten Mal kann ein Hersteller ein Produkt nicht für sich allein entwickeln, sondern ist

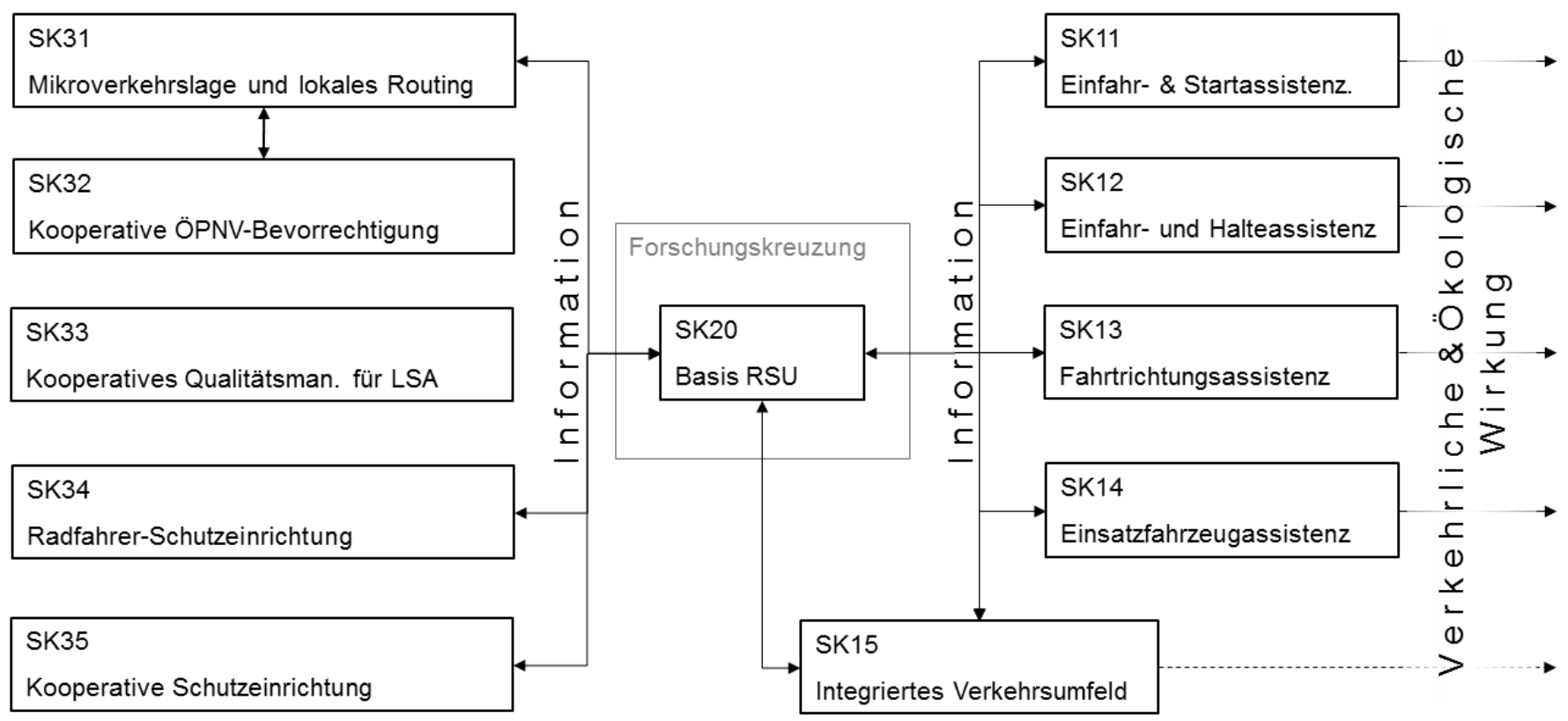

Abb. 4 Applikation der »Smarten Kreuzung« im Projekt UR:BAN

ist. Neben der Nutzung eines identischen Funkstandards kommt es auf die Nutzung identischer Nachrichtenformate an, sowie letztendlich auf die Regeln zum Erstellen der Nachrichten. Bei Fahrzeugen werden dafür zwei Formate unterschieden. Die CAM (Cooperative Awareness Message) wird von allen Teilnehmern zyklisch versendet und enthält wesentliche Informationen zur Position, Fahrzeugdynamik und Angaben zur Art des Fahrzeugs. Empfänger können sich auf diese Weise ein aktuelles Bild von Teilnehmern in ihrer Umgebung machen und die Informationen vergleichbar mit denen eines Umfeldsensors auswerten. Dabei steigen Informationstiefe und -breite - es stehen dem Empfänger im Vergleich zur heutigen Umfeldsensorik sowohl mehr Informationen über ein Objekt, als auch eine verbesserte Informationsqualität der einzelnen Signale zur Verfügung. Die Kommunikation folgt einem Sender-Empfänger-Prinzip - demnach liegt auch beim Sender von Nachrichten bereits eine gewisse Intelligenz. So können situationsabhängig zusätzliche Nachrichten verschickt werden. Diese DENM (Decentralized Environemental Notification Message) ent- auf Input, Zusammenarbeit und Test mit anderen Herstellern angewiesen. Dies belegt auch die Durchführung zahlreicher Förderprojekte in den vergangenen Jahren. Von reinen Kommunikationsversuchen haben sich die Inhalte hin zu zu funktions- und nutzerorientierten Projekten gewandelt, die Ausblicke auf die ersten Serienfunktionen bieten. Dabei waren auch früh die Infrastrukturbetreiber an Projekten beteiligt. Durch die Integration der Kommunikationskomponenten in Infrastrukturbestandteile wie Lichtsignalanlagen ergeben sich zukünftig auch für das Verkehrsmanagement von Städten neue Lösungen und Wege, Verkehr in der Stadt effizienter und schonender zu betreiben.

2.2 Förderprojekte als Schüssel zu herstellerübergreifender Interoperabilität

Das jüngste Projekt hierzu war UR:BAN (Urbaner Raum: Benutzergerechte Assistenzsysteme und Netzmanagement). An der Durchführung waren zahlreiche deutsche Automobilhersteller, Zulieferer, Universitäten und Städte 
beteiligt. Das Projekt war dabei in drei Säulen strukturiert, wobei die Säule mit dem Titel »Vernetztes Verkehrssystem « sich direkt mit den aktuellen Technologien und Funktionen zum Austausch von Informationen zur Verkehrsverbesserung beschäftigt.

Im Teilprojekt »Smarte Kreuzung« steht dabei die Entwicklung Car2X-basierter Funktionen mit WLANp im Fokus. Volkswagen hatte für dieses Teilprojekt die Leitung inne und war federführend in der Funktionsgestaltung. Die Entwicklung betraf dabei hauptsächlich Funktionen in Interaktion mit der Infrastruktur.

Im Teilprojekt »Smarte Kreuzung « werden Applikationen entwickelt, bei denen mit Hilfe von Fahrerassistenzsystemen ein effizienterer und ökologischerer Verkehrsablauf im Umfeld von Kreuzungen erzielt werden kann. Grundlage bildet dabei die Vernetzung von Straßeninfrastruktur und Verkehrsteilnehmern. Das Teilprojekt gliedert sich somit in infrastruktur- und fahrzeugseitige Applikationen. Die fahrzeugseitigen Verkehrsassistenzfunktionen (SK11-SK15) des Teilprojektes "Smarte Kreuzung " werden dabei unter der Bezeichnung »Kreuzungslotse« zusammengefasst. Die Applikationen der Verkehrsinfrastruktur (SK31-SK35) verfügen über Informationen, deren Wirkung im Wesentlichen in der Bereitstellung von Informationen besteht. Über die Intelligent Roadside Station (IRS) der Forschungskreuzung (SK20) und das »Integrierte Verkehrsumfeld« des Kreuzungslotsen (SK15) werden die Informationen den übrigen Applikationen des Kreuzungslotsen (SK11-SK14) zur Verfügung gestellt, wo dann eine direkte verkehrliche Wirkung zu verzeichnen ist (UR:BAN Projektkonsortium 2014). Abb. 4 zeigt die Vernetzung der Applikationen.

- Einfahr- und Startassistenz

Die Applikation verbessert durch eine verkehrsoptimale Längsregelung den Verkehrsfluss vor Kreuzungen mit Lichtsignalanlagen (LSA). Wie alle weiteren Applikationen ist die Einfahr- und Startassistenz für den Stadtverkehr konzipiert. Es kommt zu einer Kapazitätserhöhung am Knoten und einer Reduktion von Haltevorgängen.

\section{- Einfahr- und Halteassistenz}

Hier erfolgt auf Basis von Infrastruktur- und Umfelddaten eine Information an den Fahrer. Diese Information kann er nutzen, um assistiert die Längsführung zu verbessern.

\section{- Fahrtrichtungsassistenz}

Diese Assistenz gibt dem Fahrer detaillierte Informationen über die verkehrliche Situation an einem Knoten. Der Fahrer kann daraufhin situativ entscheiden, ob er eine kleinräumige Umleitung wählen will. Die Auswirkung von Störungen kann damit reduziert werden.

- Integriertes Verkehrsumfeld Die Applikation liefert vorausschauende Informatio- nen an den Fahrer über lokale Verkehrslage, LSA-Phasen und mögliche Gefahrensituationen. Diese werden über verschiedene Kommunikationswege ins Fahrzeug transferiert und mit der digitalen Karte verknüpft.

- Die Applikationen SK20 bis SK35 bieten Basisdienste zur Kommunikation, Verkehrslageerfassung und Informationsverteilung. Die Integration von VRU ist hier eingebettet. Die Radfahrerschutzeinrichtung erkennt Radfahrer und ihre Position wird von der Infrastrukturkomponente kommuniziert. Auf diese Weise erlangen auch die Fahrzeuge in der Umgebung Kenntnis über den Radfahrer, ohne inn selbst über einige Umfeldsensorik erfasst zu haben.

\subsection{Kommunikationsbasierte Funktionen für den} Verkehr der Zukunft

Eine Sonderstellung nimmt die Einsatzfahrzeugassistenz ein. In der Betrachtung realer Unfallszenarien von Einsatzfahrzeugen stellt sich heraus, dass Unfälle gehäuft an roten LSA vorkommen. Daraus abgeleitet wurde eine Funktion in Kooperation mit Car2X-fähiger Infrastruktur entwickelt: Die Priorisierung von Einsatzfahrzeugen an Lichtsignalanlagen durch Nutzung der schon genannten Car2X-Nachrichten. Im Rahmen der Untersuchungen wurde versucht, ein eindeutiges Bild über das Unfallgeschehen von Einsatzfahrzeugen zu erhalten. Dies ist für Deutschland nicht möglich - Unfälle werden nicht zentral erfasst, es fehlt die Kennzeichnung des Einsatzfahrzeugs in den amtlichen Unfallstatistiken. Eine fehlende Datenbasis macht auch die Auswahl geeigneter Assistenzfunktionen schwieriger. Mit Blick auf Presseberichte finden sich jedoch fast ausschließlich Meldungen über Unfälle im Querverkehr, mit der Erläuterung, der Individualverkehr hätte ein Einsatzfahrzeug »übersehen«. Doch wie kommt es dazu?

Blaulicht und Sirene sind seit den 1930er Jahren als Warneinrichtungen für Einsatzfahrzeuge bekannt. Sind beide Systeme aktiviert, genießen Einsatzfahrzeuge das Wegerecht, andere Verkehrsteilnehmer haben schnellstens freie Bahn zu schaffen um dem Einsatzfahrzeug ein ungehindertes Durchkommen zu ermöglichen. Dabei stehen zwei Herausforderungen im Raum. Der Verkehrsteilnehmer muss das Einsatzfahrzeug zunächst wahrnehmen und für sich korrekt entscheiden, ob das wahrgenommene Einsatzfahrzeug für ihn relevant ist. Dabei zeigen bestehende Studien und eigene Untersuchungen, dass Verkehrsteilnehmer aus verschiedenen Gründen bereits Probleme bei der Wahrnehmung der Warneinrichtungen haben. Das Blaulicht ist am Tag unter anderen visuellen Reizen nur schwer wahrnehmbar. Die Sirene unterliegt zwei Effekten, die eine Wahrnehmung erschweren. Die hier verwendete Tonfolge findet sich häufig in Musikstücken und kann daher im Fahrzeug leicht überhört werden. Zusätzlich erschweren Reflexionen der Schallwellen die Ortung der Quelle. Dies zeigt, dass die bestehenden 


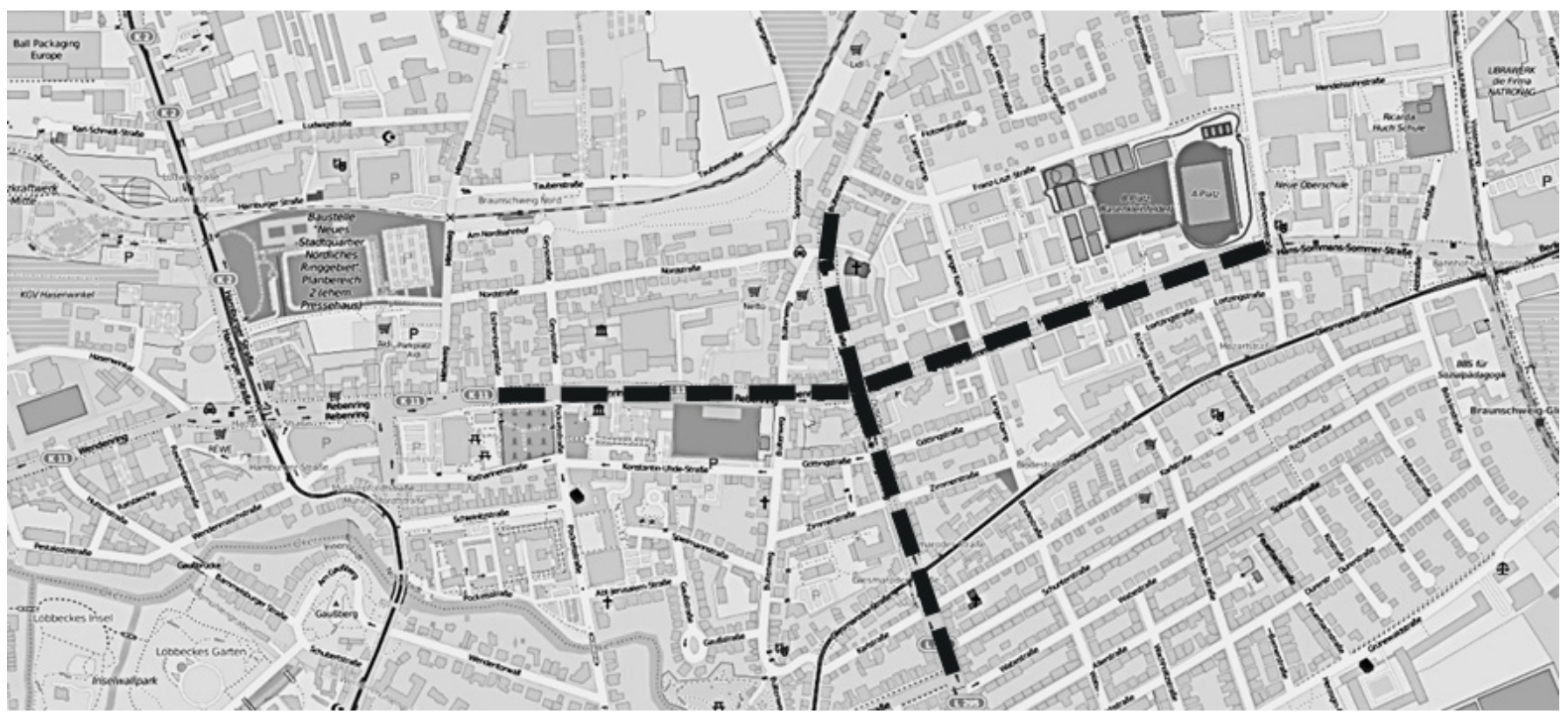

Abb. 5 Erzielbare Reichweiten am Testknoten

Warnkanäle, trotz kontinuierlicher Verbesserungen nicht optimal und nicht zeitgemäß sind. Daher werden im letzten Teil des Papers neue Vorschläge für Warneinrichtungen gemacht.

Für die Erprobung von neuartigen Applikationen wurde von der Volkswagen AG ein Volkswagen Golf VII Variant in Polizeiausführung als Versuchsträger aufgebaut. Die Kommunikation mit der IRS erfolgt durch Car2X-Kommunikation und Nutzung der Nachrichten CAM und $D E N M$. Die infrastrukturseitige IRS, sowie der Durchgriff auf das Steuergerät der LSA wurden dabei im Rahmen des Projekts vom Partner Deutsches Zentrum für Luft- und Raumfahrt ( $D L R)$ realisiert. Hierfür wurden Knotenpunkte in Braunschweig und Düsseldorf mit der Kommunikationstechnik ausgestattet.

Es wurde in beiden Feldern eine Funktionserprobung durchgeführt, bei der verschiedene Parameter untersucht wurden. Wesentlich ist dabei die Kommunikationsreichweite zwischen Fahrzeug und IRS. Zum Wechsel in ein Signalbild zur Priorisierung des Einsatzfahrzeugs benötigt das Steuergerät der Lichtsignalanlage einen gewissen Zeitraum. Die Länge dieses Zeitraums ist von Knotenpunkt zu Knotenpunkt verschieden und darüber hinaus noch abhängig von der Anfahrtsrichtung und der aktuellen Umlaufsekunde des jeweiligen Signalprogramms. Ein Zusammenhang zur Kommunikationsreichweite besteht dahingehend, dass die Anmeldung eines Einsatzfahrzeugs zur Priorisierung in einer Entfernung erfolgen muss, in der gewährleistet ist, dass das Fahrzeug während des Zeitraums zur Realisierung von grün noch mit der maximal zulässigen Geschwindigkeit auf die LSA zufahren kann, ohne die Haltelinie zu queren. Für den Anwendungsfall in Braunschweig bedeutet dies eine erforderliche Mindestreichweite von $340 \mathrm{~m}$. Entsprechend den Anforderungen der Funktion, die bereits einzelne Nachrichten als Anmeldung auswerten kann und daher nicht zwingende auf eine kontinuierliche Verbindung angewiesen ist, wurde die Entfernung als Kommunika- tionsreichweite klassifiziert, in der das jeweils erste Datenpaket vollständig empfangen wurde. Anhand dieses Datenpakets kann die IRS der LSA ein Einsatzfahrzeug als relevant identifizieren, woraufhin die Priorisierung angestoßen wird.

Die weitere Kommunikation des Einsatzfahrzeugs mit der IRS dient der Beobachtung der Fahrt während weniger Sekunden. Überquert das Einsatzfahrzeug die Haltelinie wird der Priorisierungswunsch zurück genommen. Biegt das Einsatzfahrzeug während der Zufahrt auf den Knoten bereits ab und bleibt für längere Zeit stehen oder bricht den Einsatz ab, kann der Priorisierungswunsch ebenfalls schnellstmöglich zurück genommen werden, wodurch gewährleistet ist, dass der Effekt auf den Verkehrsfluss der Nebenrichtungen minimal bleibt.

Das Fahrzeug sendet nun eine CAM mit den Merkmalen eines Einsatzfahrzeugs. Zur Identifikation werden die Daten LightBarInUse, SirenInUse und VehicleType genutzt. Die maximal erreichbaren Reichweiten für die Kommunikationsversuche in Braunschweig sind in Abb. 5 dargestellt.

Wie eingangs erwähnt, soll durch Nutzung der Einsatzfahrzeugpriorisierung die Reisezeit verringert werden und die Unfallanzahl im Kreuzungsbereich reduziert werden.

Auf Grund der Signalsteuerung der LSA ist es zu erwarten, dass die Reduzierung der Reisezeit auch abhängig von der Umlaufsekunde des LSA-Programms zum Zeitpunkt der Anmeldung des Einsatzfahrzeugs ist.

Ein reproduzierbarer Verkehr, der die Untersuchung dieser Abhängigkeit im Feld ermöglicht, ist nicht zu realisieren. Es entstand dadurch die Entscheidung, das reale Testfeld in eine Verkehrsflusssimulation zu übertragen um Vereinbarkeit von Realerprobung und Simulation zu zeigen. Eine geeignete Simulationsumgebung erlaubt dabei auch die Untersuchung des Einflusses weiterer Verkehrsanwendungen und das Heranziehen von Messgrößen, die im Realfeld so nicht zu erfassen wären. 


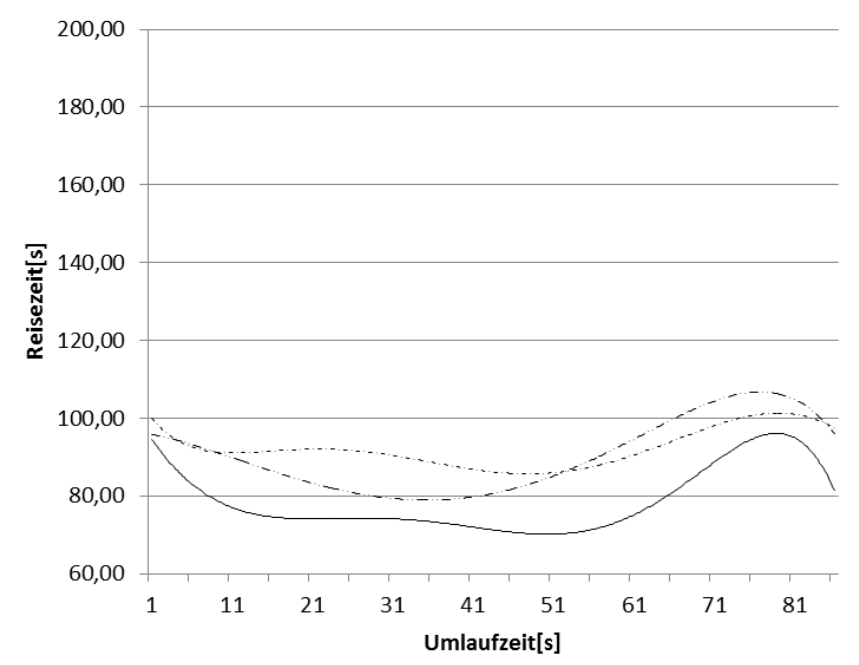

Abb. 6 Reisezeitverlauf über der Umlaufsekunde der LSA zum Zeitpunkt der Anmeldung des Priorisierungswunschs

Es ist ersichtlich, dass sowohl eine reine Priorisierung, wie auch die Reaktion des Individualverkehrs dem Einsatzfahrzeug ein schnelleres Vorankommen ermöglichen - daher ist auch die Abbildung der Reaktion des Individualverkehrs wichtig. Wird die Priorisierung für ein aktives Einsatzfahrzeug simuliert und beide Modelle kombiniert ergibt sich über die gesamte Umlaufzeit ein weiterer Zeitgewinn von durchschnittlich ca. $15 \%$, wie Abb. 6 zeigt. Die Auswirkungen des Systems auf Unfälle lassen sich durch die Simulation schwerer abbilden, da eine Bezugsgröße von Unfällen vor der Einführung des Systems fehlt. In der Simulation selbst wurden keine Unfälle mit

\section{Vorsicht Einsatzfzg.!}

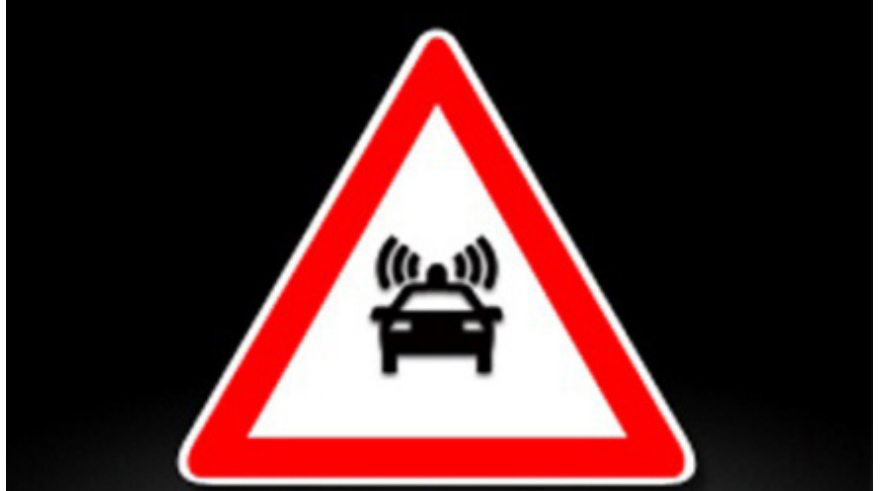

\section{OK}

\section{$\mathrm{km}$ 65324}

dem Einsatzfahrzeug festgestellt, unabhängig davon, welche Modellvariante verwendet wurde. Es ist jedoch bei optimaler Systemperformance (100\% aller Überfahrten des Einsatzfahrzeugs bei Rot werden vermieden) anzunehmen, dass das Wirkfeld bezogen auf die Insassen von Einsatzfahrzeugen in der Größenordnung von 85 \% für Schwerverletzte und Getötete liegt, wie Custalow \& Gravitz (2009) analysieren. Durch die Betrachtung der dynamischen Daten aller Fahrzeuge in der Simulation zeigt sich, dass es im Vergleich der genannten Szenarien zu homogeneren Geschwindigkeitsverläufen, zu weniger Verzögerungen und weniger starken Verzögerungswerten in Szenarien mit Priorisierung kommt. Es ist

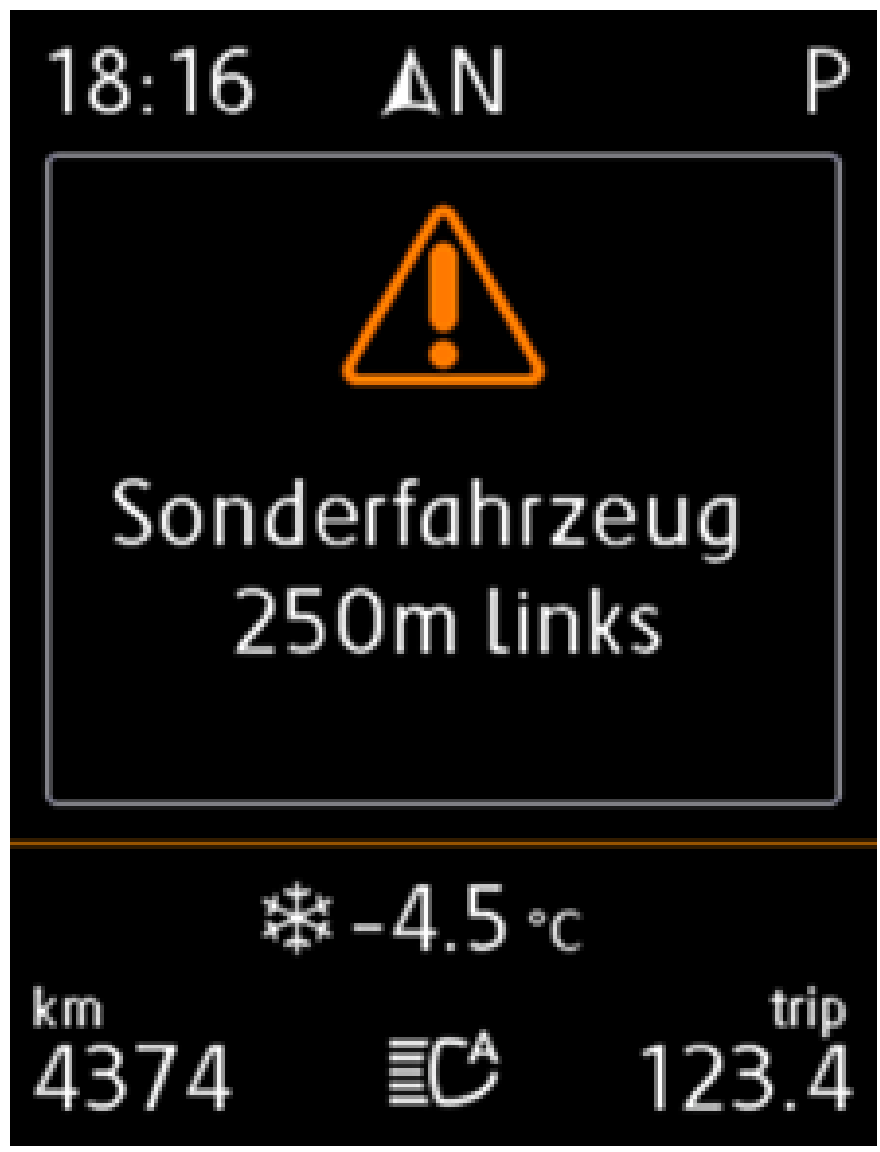

Abb. 8 Exemplarische Einsatzfahrzeugwarnung (2)

weiterhin charakteristisch, dass die Anzahl von Notbremsungen $\left(a>\left|-7 \mathrm{~m} / \mathrm{s}^{2}\right|\right)$ im Vergleich zwischen Überfahrt bei Rot und Priorisierung um $91 \%$ zurückgehen. Damit wird auch die Gefahr von Sekundärkollisionen im Längsverkehr reduziert.

\subsection{Vernetzung von Fahrzeugen untereinander}

Neben den infrastrukturbasierten Funktionen sind auch Funktionen zum direkten Austausch von Informationen zwischen Fahrzeugen besonders für Einsatzfahrzeuge realisierbar. Durch Nutzung der Car2X-Technologie wird eine gezielte Warnung anderer Verkehrsteilnehmer möglich, was im Rahmen von SK14 ebenfalls untersucht wurde. Die Warnung wird vom Empfänger nur dann an den Fahrer ausgegeben, wenn das betreffende Einsatzfahrzeug für diesen auch relevant ist. 


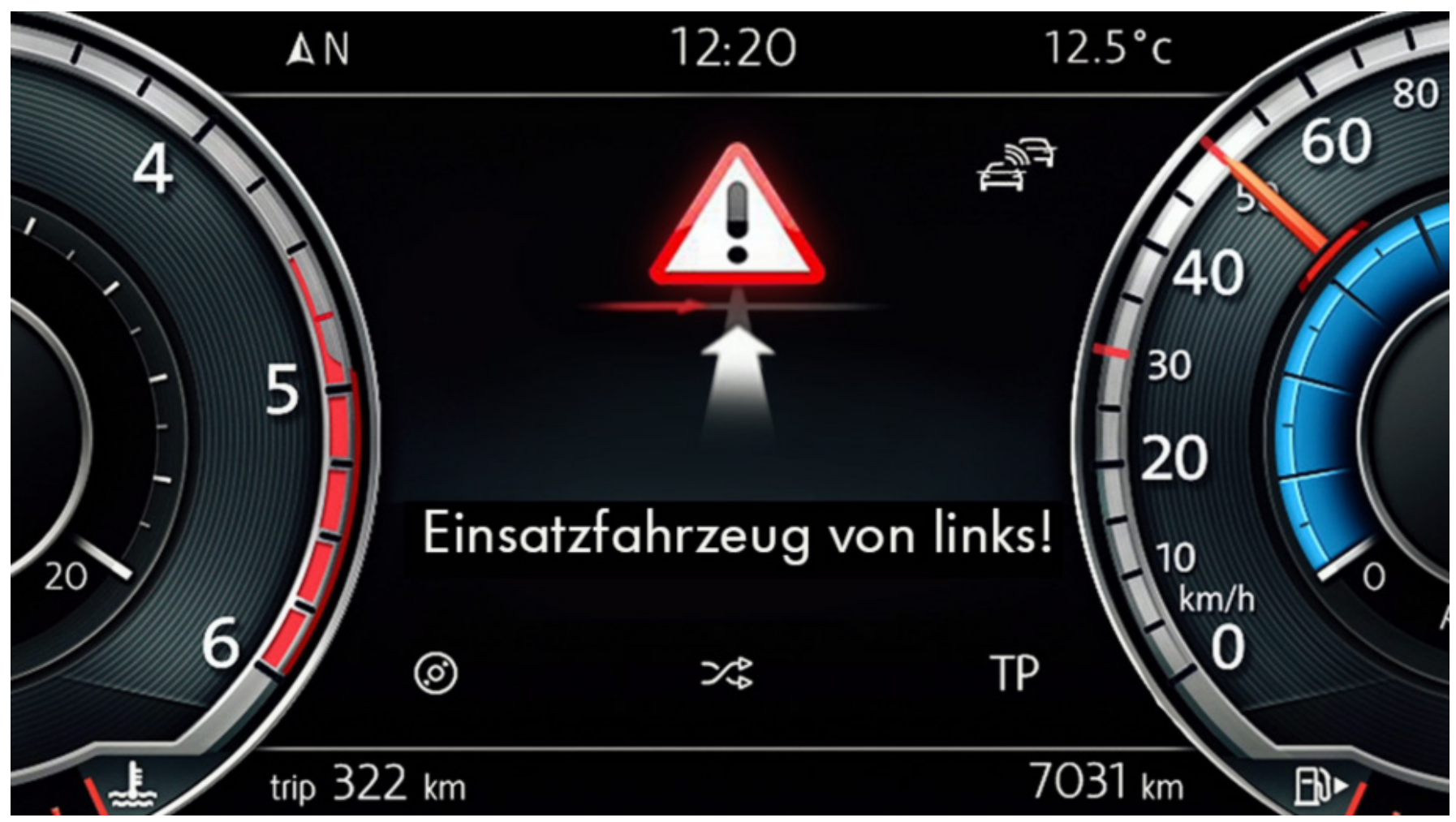

Abb.9 Exemplarische Einsatzfahrzeugwarnung (3)

Die zweite Herausforderung besteht in der korrekten Reaktion des Individualverkehrs. Hat ein Verkehrsteilnehmer ein Einsatzfahrzeug wahrgenommen und auch korrekt entschieden, dass er reagieren muss, kommt es trotzdem häufig zu falschen Reaktionen. Zum Reaktionsverhalten des Individualverkehrs auf Einsatzfahrzeuge wurde eine Befragung von Fahrern des Individualverkehrs durchgeführt. Während $96 \%$ der Probanden angeben, Blaulicht und Sirene würden ausreichen um die Aufmerksamkeit zu erwecken, geben $59 \%$ auf detaillierte Nachfrage hin an, die Richtung, aus der sich ein Einsatzfahrzeug nähert oft nicht korrekt einschätzen zu können. Noch $43 \%$ der Probanden geben an, dass sie häufig nicht wissen, ob das wahrgenommene Einsatzfahrzeug für sie relevant ist und ob es einer Reaktion bedarf. Hier zeigt sich, dass trotz eingangs nahezu vollständiger Zustimmung zur hinreichenden Wirkung von Blaulicht und Sirene, auf konkrete Nachfrage hin ein erheblicher Assistenzbedarf offensichtlich wird.

Eine Warnung ist in zwei Ausprägungen denkbar. In der ersten Ausprägung handelt es sich hierbei um eine bloße Information zur Richtung, aus der sich ein Einsatzfahrzeug nähert, verbunden mit einer Information über die Entfernung, die Information kann in ihrer Kritikalität zur Warnung eskaliert werden, mögliche Ausführungen sind exemplarisch in den Abb.7-9 dargestellt. Eine zweite Ausprägung schlagen Buchenscheit et al. (2009) vor. Die Information ist hier um einen konkreten Hinweis oder eine Handlungsempfehlung ergänzt.

\subsection{Warnstrategien und HMI-Gestaltung}

Im Kontext von Informationen an den Fahrer, muss besonders die Gestaltung des Informationspfades be- rücksichtigt werden, um dem Fahrer eine optimale Verarbeitbarkeit zu gewährleisten. Auf Basis dieser Verarbeitbarkeit ist eine schnelle und korrekte Reaktion des Fahrers optimal möglich. Ziel ist es, beim Fahrer ein Situationsbewusstsein zu schaffen, dass inn in die Lage versetzt eine der Situation angemessene Entscheidung zu treffen. Es stehen dazu der akustische, optische und haptische Informationskanal zur Verfügung, wobei sich bei Meldungen zu Umfeldinformationen der akustische und optische Informationskanal weitgehend etabliert haben. Im Fahrzeug stehen zu diesem Zweck ein elektronischer Gong für akustische Signale sowie verschiedene Displays zur Verfügung.

Darüber hinaus gehende Informationen, wie eine Handlungsempfehlung an den Fahrer sind jedoch nur mit vollständiger Erfassung der Fahrzeugumgebung und unter Berücksichtigung des Verhaltens anderer Verkehrsteilnehmer möglich. Handlungsempfehlungen können eine Brückentechnologie hin zum Automatischen Fahren bilden, sie stehen aber nicht mehr im Fokus dieses Papers.

Das Zusammenwirken von Infrastrukturapplikationen und einer gezielten Information an den Fahrer kann damit dazu beitragen, Situationen zu entschärfen und einen Beitrag zum sichereren Fahren in der Stadt zu leisten. Erste Funktionen, die Mehrwerte bieten, sind auf Basis standardisierter Car2X-Kommunikation entwickelt und erprobt worden. Sie bieten herstellerübergreifenden Informationsaustausch und zeigen in Simulation und realem Einsatz positive Ergebnisse. Car2X-Kommunikation wird so zu einer leistungsstarken neuen Technologie zur Umfelderfassung und Möglichkeit mit der Infrastruktur in Austausch zu treten. 


\section{Literatur}

ADAC (2015) Zur Sache: Autobahn-Tempolimit. https://www. adac.de/_mmm/pdf/rv_tempolimit_sp_1015_83113.pdf. Accessed 20 Jan 2016

Bakaba JE, Kühn M (2009) Bekämpfung von Baumunfällen auf Landstraßen. Unfallforschung kompakt, vol 13. Gesamtverband der Deutschen Versicherungswirtschaft: Unfallforschung der Versicherer, Berlin

Buchenscheit A, Schaub F, Kargl F, Weber M (2009) A VANET-based emergency vehicle warning system. In: Proc IEEE Vehicular Networking Conference (VNC), 28-30 Oct 2009, Tokyo, ISBN: 978-1-4244-5685-7, pp 1-8. doi: 10.1109/ VNC.2009.5416384

Custalow CB, Gravitz CS (2009) Emergency medical vehicle collisions and potential for preventive intervention. Prehosp Emerg Care 8(2):175-184. doi: 10.1080/31270300279X

Fildes B, Keall M, Bos N, Lie A, Page Y, Pastor C, Pennisi L, Rizzi M, Thomas P, Tingvall C (2015) Effectiveness of low speed autonomous emergency braking in real-world rear-end crashes. Accid Anal Prev 81:24-29. doi: 10.1016/j.aap.2015.03.029

Pion O, Henze R, Küçükay F (2012) Fingerprint des Fahrers zur Adaption von Assistenzsystemen. In: Goltz U, Magnor M, Appelrath H, Matthies HK, et al. (eds) Informatik 2012. Lecture Notes in Informatics, P-208. Gesellschaft für Informatik, Bonn, ISBN: 978-3-88579-602-2, pp 833-842

Radke S (2013) Verkehr in Zahlen 2013/14, 42nd edn. Bundesministerium für Verkehr, Bau und Stadtentwicklung; Deutsches Institut für Wirtschaftsforschung; Deutscher Verkehrsverlag, Hamburg. ISBN: 978-3-87154-493-4

Statistisches Bundesamt (2015) Getötete bei Straßenverkehrsunfällen nach Beteiligungsart und Unfallort 2014. https:// www.destatis.de/DE/ZahlenFakten/Wirtschaftsbereiche/ TransportVerkehr/_Grafik/Verkehrsunfaelle_GetoeteteBeteiligungsart.png?__blob=poster. Accessed 21 Jan 2016

Thatham Research (2015) Golf Drives Down Personal Injury. https://www.thatcham.org/news-and-events/news-andpress-releases-reader/items/golf-drives-down-personal-injury. Accessed 16 Jan 2016

UR:BAN Projektkonsortium (2014) Meilensteinbericht MS 2. Projektbericht

\section{Autoren}

André Leschke

Volkswagen AG

Wolfsburg

Florian Weinert

Volkswagen AG

Wolfsburg 